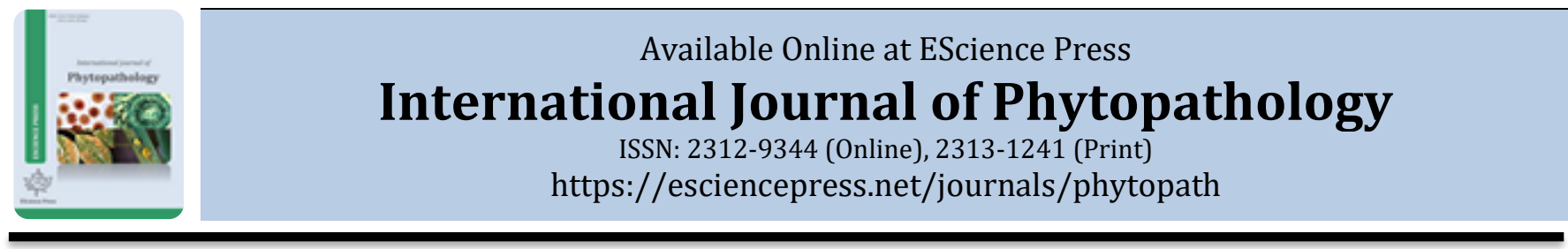

\title{
THREE COLLETOTRICHUM SPECIES RESPONSIBLE FOR ANTHRACNOSE ON SYNSEPALUM DULCIFICUM (MIRACLE FRUIT)
}

aHanh. H. Truong, bToyozo Sato, aSeiju Ishikawa, aAyaka Minoshima, aTakeaki Nishimura, aYuuri Hirooka* ${ }^{a}$ Department of Clinical Plant Science, Hosei University, Koganei, Tokyo, Japan.

${ }^{b}$ Genetic Resources Center, National Agriculture and Food Organization (NARO) 2-1-2, Kannondai, Tsukuba, Ibaraki, Japan.

*Corresponding Author Email: yuurihirooka@hosei.ac.jp

A B S T R A C T

In 2016 and 2017, fruit rot and two different leaf diseases (leaf spot and leaf blight) were found on Synsepalum dulcificum (miracle fruit) in Tokyo, Kanagawa and Kagoshima prefectures of Japan. From the lesions, abundant conidial masses and acervuli of three Colletotrichum species, two of which produced sexual state, were observed. We conducted a pathogenicity assay using these Colletotrichum species on healthy fruits and leaves of $S$. dulcificum. Our artificial inoculation tests showed symptoms of disease on tested fruit and leaf and indicated all three Colletotrichum species as causal agents of anthracnose on S. dulcificum. Based on morphological characters and molecular phylogenetic analyses using ITS, GAPDH, ACT, CAL and TUB2 loci, these species were identified as Colletotrichum aenigma (MAFF 246750), $C$. siamense (MAFF 246751) and C. karstii (MAFF 245966). They have been previously reported as plant pathogenic fungi elsewhere in the world. This is the first report of fruit rot, leaf blight and leaf spot on S. dulcificum caused by these three Colletotrichum species.

Keywords: Colletotrichum aenigma, C. karstii, C. siamense, fruit rot, leaf anthracnose, miracle fruit, molecular phylogeny.

\section{INTRODUCTION}

The genus Colletotrichum is one of the most important plant pathogenic fungal groups in the world. The genus causes diseases on a wide variety of woody and herbaceous plants and is the principal cause of serious plant diseases especially in tropical and the sub-tropical regions (Da Silva and Michereff, 2013; De Silva et al., 2016; Lima et al., 2013). Colletotrichum has recently been voted as the world's eighth most economically important fungal pathogens, based on perceived scientific and economic criteria (Dean et al., 2012). Interestingly, previous studies showed that one species of Colletotrichum can cause disease on multiple host plants, and multiple species can jointly infect a single host (Nguyen et al., 2009; Sharma and Shenoy, 2016). According to Rojas et al. (2010), Colletotrichum spp. are the principal cause of damping-off, leaf spot, seedling blight as well as pre- and post-harvest fruit rot. These disease symptoms appear in developing and mature plant tissues of diverse hosts such as fruit, vegetables and ornamental plants (Da Silva and Michereff, 2013; Zivkovic et al., 2010). For the purposes of plant quarantine,
Colletotrichum-infected commodities are not suitable for import or export due to the potential revenue loss (Sharma and Shenoy, 2016). Precise identification plays an important role for understanding the epidemiology of Colletotrichum species and developing effective disease control methods. Yokosawa et al. (2017), for instance, mentioned that the different levels of resistance to several fungicides was observed among members of the Colletotrichum gloeosporioides species complex. Traditional identification systems in Colletotrichum were mainly based on morphological and cultural characters as well as host association (Alizadeh et al., 2015; Lima et al., 2013). However, morphology alone is inadequate to provide sufficient and informative characters for an accurate identification (Alizadeh et al., 2015). Therefore, molecular analyses with multiple loci coupled with morphological characters is now the preferred method for precise identification of the Colletotrichum species (Cai et al., 2009).

Synsepalum dulcificum (Sapotaceae) is commonly known as miracle fruit, miraculous berry or sweet berry (Akinmoladun, 2016; Shi et al., 2016). This plant originates 
from tropical West Africa (Akinmoladun, 2016; Rodrigues et al., 2016; Shi et al., 2016). It has subsequently been treated as an important plant because of an active compound in the fruit called miraculin. Miraculin is a single polypeptide chain, which is used to modify taste in food and to control obesity (Akinmoladun, 2016).

In 2016 and 2017, we found fruit rot and two different leaf symptoms of $S$. dulcificum in Tokyo, Kanagawa and Kagoshima prefectures, Japan (Figure 1). The fruit rot was first observed in a greenhouse of the botanical garden in Kanagawa prefecture. During our research, the disease was constantly observed to cause damage to the host plant. From microscopic examination of plant symptoms, conidial masses and acervuli of the genus Colletotrichum were prominent. Two leaf symptoms, leaf blight and leaf spot, were observed in Tokyo and Kagoshima prefecture respectively. An initial symptom of leaf blight was small lesion at the tip of the leaf, and the lesion then developed and increased in size towards the petiole. Morphological features of the genus Colletotrichum such as conidial masses and setae on acervuli, were observed from the symptoms. The leaf spot was first started as tiny black dots at leaf margin. The black dots then developed and produced big spots and chlorosis areas encompassed by a dark brown line. Both diseased leaves were eventually defoliated.
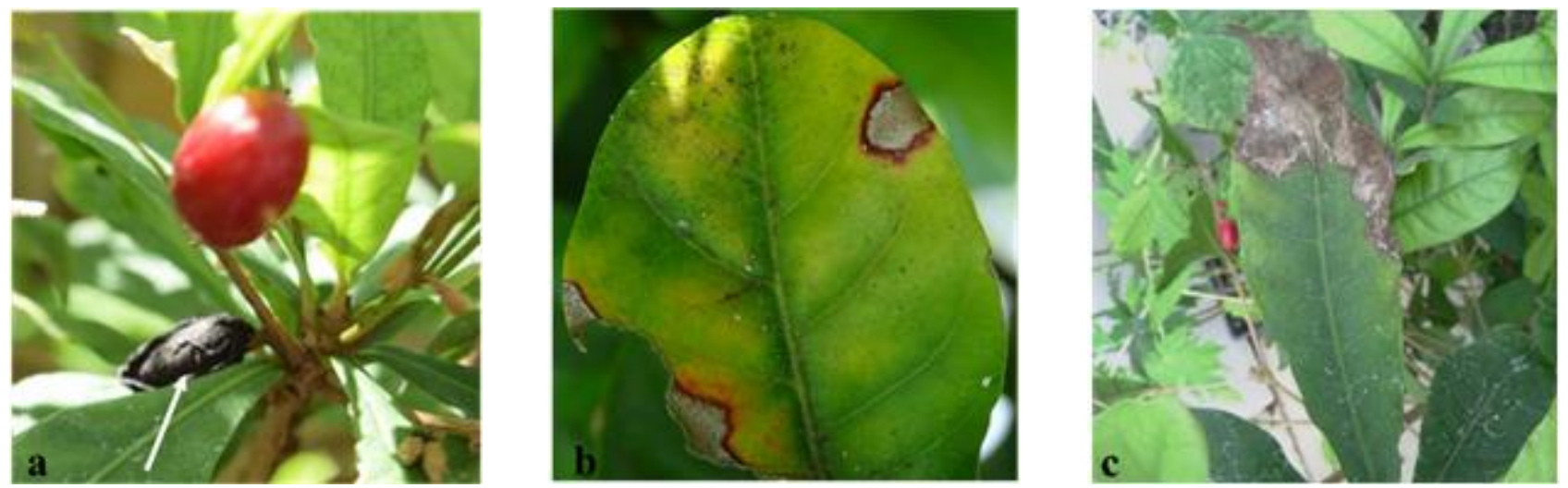

Figure 1. Original symptoms caused by Colletotrichum spp. on S. dulcificum. a: Fruit rot (white arrow). b: Leaf spot. c: Leaf blight.

Although S. dulcificum is a notable tropical plant, there have not been many studies focusing on its pathology until now. To the best of our knowledge, the only leaf disease reported on $S$. dulcificum was caused by Pestalotiopsis synsepali (Chen et al., 2002). Damm et al. (2012) found C. karstii on leaf of $S$. dulcificum, but its pathogenicity on $S$. dulcificum has been unknown. The aims of this study were: (1) to identify these three Colletotrichum species causing of anthracnose on $S$. dulcificum based on morphology and molecular analyses; (2) to determine their pathogenicity to S. dulcificum.

\section{MATERIALS AND METHODS}

Sampling and fungal isolation: Fruit rot of S. dulcificum was observed in a greenhouse of the botanical garden located Kamakura, Kanagawa, in 2016. From its symptom, conidial masses were collected and suspended in sterile water. The prepared conidial suspension was then spread over the surface of water agar (WA). After 24 hours, a single germinating spore was transferred onto Difco $^{\mathrm{TM}}$ potato dextrose agar (PDA; Detroit, MI, USA).
Two different leaf symptoms of $S$. dulcificum were determined in different regions. Leaf spot was observed in a fruit garden at Tanegashima island, Kagoshima in 2016 while leaf blight was found in a greenhouse, in Tokyo in 2017. The aforementioned isolation method was employed both for leaf spot and blight diseases. The isolates from fruit rot (MAFF 246750), leaf blight (MAFF 246751) and leaf spot (MAFF 245966) were obtained and preserved at the Genebank, National Agriculture and Food Research Organization (NARO), Tsukuba, Ibaraki, Japan.

Pathogenicity assay: Colletotrichum isolates were grown on PDA for seven days at $25{ }^{\circ} \mathrm{C}$. Spores were harvested by using $10 \mathrm{ml}$ of sterilized distilled water to pour into the cultures, and the water was gently swirled to dislodge the conidia. Conidial density was adjusted to get $10^{6}$ conidia/ml by using a haemocytometer (Prihastuti et al., 2009).

The wound/non-wound treatments for the pathogenicity assay were performed on healthy fruits 
and leaves of potted S. dulcificum seedlings. The wounds were made by pricking the surface of the miracle fruits or leaves with a sterilized needle. The conidial suspension was sprayed on the wounded/non-wounded fruits and leaves, while sterilized distilled water was used as control. The inoculated and non-inoculated fruits and leaves were covered by plastic bags and then placed in a greenhouse under $25-30{ }^{\circ} \mathrm{C}$. Plastic bags were removed after 48 hours. Disease symptoms such as fruit rot, leaf blight and leaf spot were observed after seven days. These experiments were performed with three replicates for each isolate.

Morphological identification: These Colletotrichum isolates growing on PDA were used for morphological examination. Morphological and cultural characters such as shape and size of conidia and appressoria, and presence or absence of setae were observed on PDA plate growing at $28{ }^{\circ} \mathrm{C}$ after one week. Shape and size of 30 conidia from each isolate were evaluated. Images under a stereo microscope (Olympus, Tokyo, Japan) and a compound microscope (Olympus, Tokyo, Japan) were captured with a digital camera (Olympus DP21, Tokyo, Japan). Conidial size was calculated by using 'imageJ' software (free download available at http://rsbweb.nih.gov/ij/).

Appressoria were produced by using a slide culture technique. A $10 \mathrm{~mm}^{2}$ square block of Synthetic Lownutrient Agar (SNA) was placed on a sterile slide glass that was kept in an empty petri dish, and the edge of the agar blocks was inoculated on one side with mycelium. The inoculated agar block was covered by a sterile coverslip (Lima et al., 2013). Seven days after inoculation, shape and size of 30 appressoria from each isolate were measured.

DNA extraction, sequencing, and analysis: Our obtained cultures were grown on PDA for seven days, and mycelia were scraped from the colony surfaces. Genomic DNA was extracted from the harvested mycelia using UltraClean ${ }^{\circledR}$ Microbial DNA Isolation Kit (MOBIO, Laboratories, Inc., California, USA) based on the instruction of the manufacturer. Sequences were obtained from five loci, namely internal transcribed spacer (ITS), glyceraldehyde-3-phosphate dehydrogenase $(G A P D H)$, actin $(A C T)$, calmodulin $(C A L)$, and $\beta$-tubulin 2 (TUB2). The loci were amplified and sequenced using the primer pairs: ITS-1/ITS-4 for ITS (Gardes and Bruns, 1993), GDF/GDR for GAPDH
(Guerber et al., 2003), ACT-521F/ACT-783R for ACT (Carbone and Kohn, 1999), CL1C/CL2C for CAL (O'Donnell et al., 2000) and T1/T2 for TUB2 (O'Donnell and Cigelnik, 1997).

The PCR conditions for ITS amplification were 4 minutes at $95{ }^{\circ} \mathrm{C}$; then 35 cycles of $95^{\circ} \mathrm{C}$ for 30 seconds, $52^{\circ} \mathrm{C}$ for 30 seconds, $72^{\circ} \mathrm{C}$ for 45 seconds and final extension at 72 ${ }^{\circ} \mathrm{C}$ for 7 minutes. Different annealing temperatures were used for other loci: $G A P D H$ at $60^{\circ} \mathrm{C}$; $A C T$ at $58{ }^{\circ} \mathrm{C}$; $C A L$ at $59{ }^{\circ} \mathrm{C}$ and TUB2 at $55{ }^{\circ} \mathrm{C}$ (Weir et al., 2012). All PCR amplification products were separated by using electrophoresis in $0.7 \%$ agarose gel in 1.0x Tris-acetate acid EDTA (TAE) buffer, and pictures were taken under UV light after staining the gel with ethidium bromide for 10 to 15 minutes. PCR products were purified using ExoSap-IT PCR Clean-up kit (GE Healthcare Life Science, Buckinghamshire, UK), following the manufacturer's instructions. DNA sequencing was performed by $3130 \mathrm{xl}$ Genetic Analyzers (Applied Biosystems, California, USA) using BigDye v.3.1 chemistry (Life Technologies, California, USA).

Sequence queries were submitted to the BLAST search engine of NCBI GeneBank (https://www.ncbi.nlm. nih.gov/). Phylogenetic trees were constructed using data from this study with other sequences extracted from GeneBank (Table 1 and 2). The consensus sequences of each region were aligned using Mesquite version 3.2 (Maddison, 2017). All ambiguously aligned regions were excluded from the analyses by eyes. The analyses were first performed on ITS region. Phylogenetic analyses were performed on the combined dataset of five mentioned loci by maximum likelihood (ML) method using RAxML (Version 0.6.0). Branch and branch node support was determined using 100 bootstrap replicates (Stamatakis et al., 2008).

\section{RESULTS}

Pathogenicity assay: The pathogenicity assay showed that MAFF 246750 isolated from fruit rot produced dark brown lesions around wounded area (Figure 2). Seven days after inoculation, all tested fruits developed the symptoms of fruit rot. From the symptoms, the inoculated fungus was re-isolated. Non-wounded fruits did not show any symptoms.

The assay conducted on leaves indicated that both isolates, MAFF 245966 and MAFF 246751, were able to cause leaf diseases on miracle fruit. 
Table 1. Isolates in the phylogenetic analysis of the Colletotrichum gloeosporioides species complex.

\begin{tabular}{|c|c|c|c|c|c|c|}
\hline \multirow{2}{*}{ Species } & \multirow{2}{*}{ Accession number* } & \multicolumn{5}{|c|}{ GenBank number } \\
\hline & & ITS & GAPDH & $C A L$ & $A C T$ & TUB2 \\
\hline C. aenigma & ICMP 18608* & JX010244 & JX010044 & JX009683 & JX009443 & JX010389 \\
\hline C. aenigma & MAFF 246750 & LC412412 & LC412415 & LC412414 & LC412413 & LC412416 \\
\hline C. aeschynomenes & ICMP $17673^{*}$ & JX010176 & JX009930 & JX009721 & JX009483 & JX010392 \\
\hline C. alatae & CBS 304.67* & JX010190 & JX009990 & JX009738 & JX009471 & JX010383 \\
\hline C. alienum & ICMP 12071* & JX010251 & JX010028 & JX009654 & JX009572 & JX010411 \\
\hline C. aotearoa & ICMP 18537* & JX010205 & JX010005 & JX009611 & JX009564 & JX010420 \\
\hline C. asianum & ICMP 18580* & FJ972612 & JX010053 & FJ917506 & JX009584 & JX010406 \\
\hline C. boninense & CBS $123755^{*}$ & JQ005153 & JQ005240 & JQ005674 & JQ005501 & JQ005588 \\
\hline C. changpingense & MFLUCC 15-0022 & KР683152 & KP852469 & - & KP683093 & KP852490 \\
\hline C. clidemiae & ICMP 18658* & JX010265 & JX009989 & JX009645 & JX009537 & JX010438 \\
\hline C. conoides & CGMCC 3.17615* & KР890168 & KP890162 & KP890150 & KP890144 & КР890174 \\
\hline C. cordylinicola & MFLUCC 090551* & JX010226 & JX009975 & HM470238 & HM470235 & JX010440 \\
\hline C. endophytica & MFLUCC 13-0418* & KC633854 & KC832854 & KC810018 & KF306258 & - \\
\hline C. fructicola & ICMP 18581* & JX010165 & JX010033 & FJ917508 & FJ907426 & JX010405 \\
\hline C. fructicola (syn. C. ignotum) & CBS $125397\left(^{*}\right)$ & JX010173 & JX010032 & JX009674 & JX009581 & JX010409 \\
\hline C. fructicola (syn. Glomerrella cingulata var. minor) & CBS $238.49\left(^{*}\right)$ & JX010181 & JX009923 & JX009671 & JX009495 & JX010400 \\
\hline C. fructivorum & CBS $133125^{*}$ & JX145145 & - & - & - & JX145196 \\
\hline C. gloeosporioides & IMI 356878* & JX010152 & JX010056 & JX009731 & JX009531 & JX010445 \\
\hline C. grevilleae & CBS $132879^{*}$ & КС297078 & KC297010 & KC296963 & KC297056 & KC296941 \\
\hline C. grossum & CGMCC 3.17614* & KP890165 & KP890159 & KР890147 & KP890141 & KP890171 \\
\hline C. hebeiense & MFLUCC13-0726* & KF156863 & KF377495 & - & KF377523 & KF288975 \\
\hline C. henanense & CGMCC $3.17354^{*}$ & KJ955109 & KJ954810 & KJ954662 & KM023257 & KJ955257 \\
\hline C. hippeastri & CBS 241.78 & JX010293 & JX009932 & JX009740 & JX009485 & JX009838 \\
\hline C. horii & NBRC $7478^{*}$ & GQ329690 & GQ329681 & JX009604 & JX009438 & JX010450 \\
\hline C. jiangxiense & CGMCC 3.17363* & KJ955201 & KJ954902 & KJ954752 & KJ954471 & KJ955348 \\
\hline C. kahawae subsp. ciggaro & ICMP 18539* & JX010230 & JX009966 & JX009635 & JX009523 & JX010434 \\
\hline $\begin{array}{l}\text { C. kahawae subsp. ciggaro (syn. Glomerella cingulata var. } \\
\text { migrans) }\end{array}$ & CBS $237.49\left(^{*}\right)$ & JX010238 & JX010042 & JX009636 & JX009450 & JX010432 \\
\hline $\begin{array}{l}\text { C. kahawae subsp. ciggaro (syn. Glomerella rufomaculans } \\
\text { var. vaccinii) }\end{array}$ & CBS $124.22\left(^{*}\right)$ & JX010228 & JX009950 & JX009744 & JX009536 & JX010433 \\
\hline
\end{tabular}




\begin{tabular}{|c|c|c|c|c|c|c|}
\hline C. kahawae subsp. kahawae & IMI 319418* & JX010231 & JX010012 & JX009642 & JX009452 & JX010444 \\
\hline C. musae & CBS $116870^{*}$ & JX010146 & JX010050 & JX009742 & JX009433 & HQ596280 \\
\hline C. nupharicola & CBS $470.96^{*}$ & JX010187 & JX009972 & JX009663 & JX009437 & JX010398 \\
\hline C. proteae & CBS $132882^{*}$ & KC297079 & KC297009 & KC296960 & KC296940 & KC297101 \\
\hline C. psidii & CBS $145.29 *$ & JX010219 & JX009967 & JX009743 & JX009515 & JX010443 \\
\hline C. queenslandicum & ICMP 1778* & JX010276 & JX009934 & JX009691 & JX009447 & JX010414 \\
\hline C. rhexiae & CBS $133134^{*}$ & JX145128 & - & - & - & JX145179 \\
\hline C. salsolae & ICMP 19051* & JX010242 & JX009916 & JX009696 & JX009562 & JX010403 \\
\hline C. siamense & ICMP $18578^{*}$ & JX010171 & JX009924 & FJ917505 & FJ907423 & JX010404 \\
\hline C. siamense & MAFF 246751 & LC412417 & LC412420 & LC412419 & LC412418 & LC412421 \\
\hline C. siamense (syn. C. hymenocallidis) & CBS $125378\left(^{*}\right)$ & JX010278 & JX010019 & JX009709 & GQ856775 & JX010410 \\
\hline C. siamense (syn. jasmini- sambac) & CBS $130420\left({ }^{*}\right)$ & HM131511 & HM131497 & JX009713 & HM131507 & JX010415 \\
\hline C. syzygicola & MFLUCC 10-0624* & KF242094 & KF242156 & KF254859 & KF157801 & KF254880 \\
\hline C. temperatum & CBS $133122^{*}$ & JX145159 & - & - & - & JX145211 \\
\hline C. theobromicola & CBS $124945^{*}$ & JX010294 & JX010006 & JX009591 & JX009444 & JX010447 \\
\hline C. theobromicola (syn. C. fragariae) & CBS $142.31\left(^{*}\right)$ & JX010286 & JX010024 & JX009592 & JX009516 & JX010373 \\
\hline C. theobromicola (syn. C. gloeosporioides f. stylosanthis) & MUCL $42294(*)$ & JX010289 & JX009962 & JX009597 & JX009575 & JX010380 \\
\hline C. $t i$ & ICMP 4832* & JX010269 & JX009952 & JX009649 & JX009520 & JX010442 \\
\hline C. tropicale & CBS $124949^{*}$ & JX010264 & JX010007 & JX009719 & JX009489 & JX010407 \\
\hline C. viniferum & GZAAS 5.08601* & JN412804 & JN412798 & JQ309639 & JN412795 & JN412813 \\
\hline C. wuxiense & CGMCC 3.17894* & KU251591 & KU252045 & KU251833 & KU251672 & KU252200 \\
\hline C. xanthorrhoeae & BRIP 45094* & JX010261 & JX009927 & JX009653 & JX009478 & JX010448 \\
\hline Glomerella cingulata "f.sp. camelliae" & ICMP 10646 & JX010225 & JX009993 & JX009629 & JX009563 & JX010437 \\
\hline
\end{tabular}

*= ex-type culture, $\left.\quad{ }^{*}\right)=$ ex-type culture of synonymized taxon

BRIP = Queensland Plant Pathology Herbarium (Australia); CBS: Culture collection of the Westerdijk Fungal Biodiversity Instit ute, Utrecht, The Netherlands; CGMCC: Chinese General Microbiological Culture Collection Center, Beijing, China; GZAAS: Guizhou Academy of Agriculture Science, Guizhou Province, China; ICMP: International Collection of Microorganisms from Plants, Auckland, New Zealand; IMI = CABI Genetic Resource Collection (UK); MAFF: Genebank Project, the Genetic Resources Center, NARO (National Agriculture and Food Research Organization), Tsukuba, Japan; MFLUCC = Mae Fah Luang University Culture Collection (Thailand); MUCL = Belgian Coordinated Collections of Microorganisms, (agro) industrial fungi \& yeasts (Belgium); NBRC = Biological Resource Center, National Institute of Technology and Evaluation (Japan); ITS: internal transcribed spacers and intervening 5.8S nrDNA; GAPDH: partial glycealdahyde3-phosphate dehydrogenase gen; CAL: partial calmodulin; ACT: partial actin gene; TUB2: partial beta-tubulin gene. Sequences generated in this study are emphasized in bold. 
Table 2. Isolates in the phylogenetic analysis of the Colletotrichum boniense species complex.

\begin{tabular}{|c|c|c|c|c|c|c|}
\hline \multirow{2}{*}{ Species } & \multirow{2}{*}{ Accession number* } & \multicolumn{5}{|c|}{ GenBank number } \\
\hline & & ITS & $G A P D H$ & $C A L$ & $A C T$ & TUB2 \\
\hline C. annellatum & CBS 129826* & JQ005222 & JQ005309 & JQ005743 & JQ005570 & JQ005656 \\
\hline C. beeveri & ICMP 18594* & JQ005171 & JQ005258 & JQ005692 & JQ005519 & JQ005605 \\
\hline C. boninense & MAFF 305972* & JQ005153 & JQ005240 & JQ005674 & JQ005501 & JQ005588 \\
\hline C. brasiliense & ICMP 18607 & JQ005235 & JQ005322 & JQ005756 & JQ005583 & JQ005669 \\
\hline C. brassicicola & CBS 101059 & JQ005172 & JQ005259 & JQ005693 & JQ005520 & JQ005606 \\
\hline C. camelliae-japonicae & CGMCC 3.18118* & KX853165 & KX893584 & - & KX893576 & KX893580 \\
\hline C. citricola & CBS 134228* & KC293576 & KC293736 & KC293696 & KC293616 & KC293656 \\
\hline C. colombiense & CBS 129818 & JQ005174 & JQ005261 & JQ005695 & JQ005522 & JQ005608 \\
\hline C. constrictum & ICMP 12936 & JQ005237 & JQ005324 & JQ005758 & JQ005585 & JQ005671 \\
\hline C. cymbidiicola & IMI 347923* & JQ005166 & JQ005253 & JQ005687 & JQ005514 & JQ005600 \\
\hline C. dacrycarpi & ICMP 19107* & JQ005236 & JQ005323 & JQ005757 & JQ005584 & JQ005670 \\
\hline C. gloeosporioides & STE-U 4295* & JQ005152 & JQ005239 & JQ005673 & JQ005500 & JQ005587 \\
\hline C. hippeastri & CBS 241.78 & JQ005232 & JQ005319 & JQ005753 & JQ005580 & JQ005666 \\
\hline C. karstii & CBS 128552 & JQ005188 & JQ005275 & JQ005709 & JQ005536 & JQ005622 \\
\hline C. karstii & CORCG6 (CGMCC 3.14194) & HM585409 & HM585391 & HM582013 & HM581995 & HM585428 \\
\hline C. karstii & MAFF 245966 & LC412407 & LC412410 & LC412409 & LC412408 & LC412411 \\
\hline C. novae-zelandiae & ICMP $12944^{*}$ & JQ005228 & JQ005315 & JQ005749 & JQ005576 & JQ005662 \\
\hline C. oncidii & CBS 129828* & JQ005169 & JQ005256 & JQ005690 & JQ005517 & JQ005603 \\
\hline C. parsonsiae & ICMP $18590^{*}$ & JQ005233 & JQ005320 & JQ005754 & JQ005581 & JQ005667 \\
\hline C. petchii & CBS $378.94^{*}$ & JQ005223 & JQ005310 & JQ005744 & JQ005571 & JQ005657 \\
\hline C. phyllanthi & MACS $271^{*}$ & JQ005221 & JQ005308 & JQ005742 & JQ005569 & JQ005655 \\
\hline C. torulosum & ICMP 18586* & JQ005164 & JQ005251 & JQ005685 & JQ005512 & JQ005598 \\
\hline
\end{tabular}

* = ex-type culture,

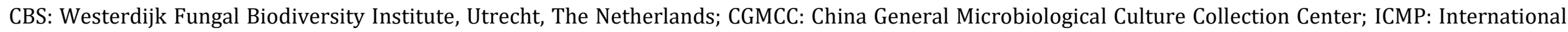

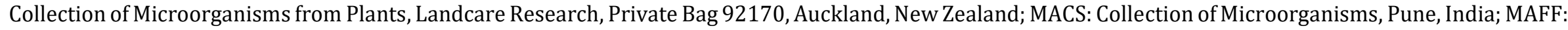

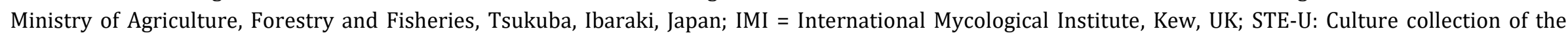

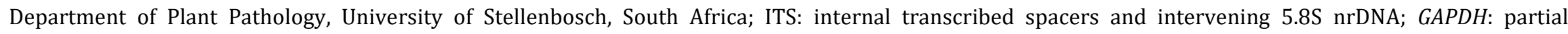

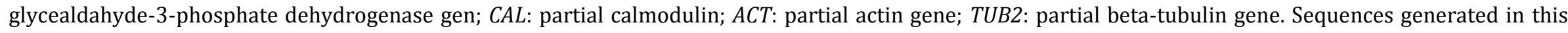
study are emphasized in bold. 

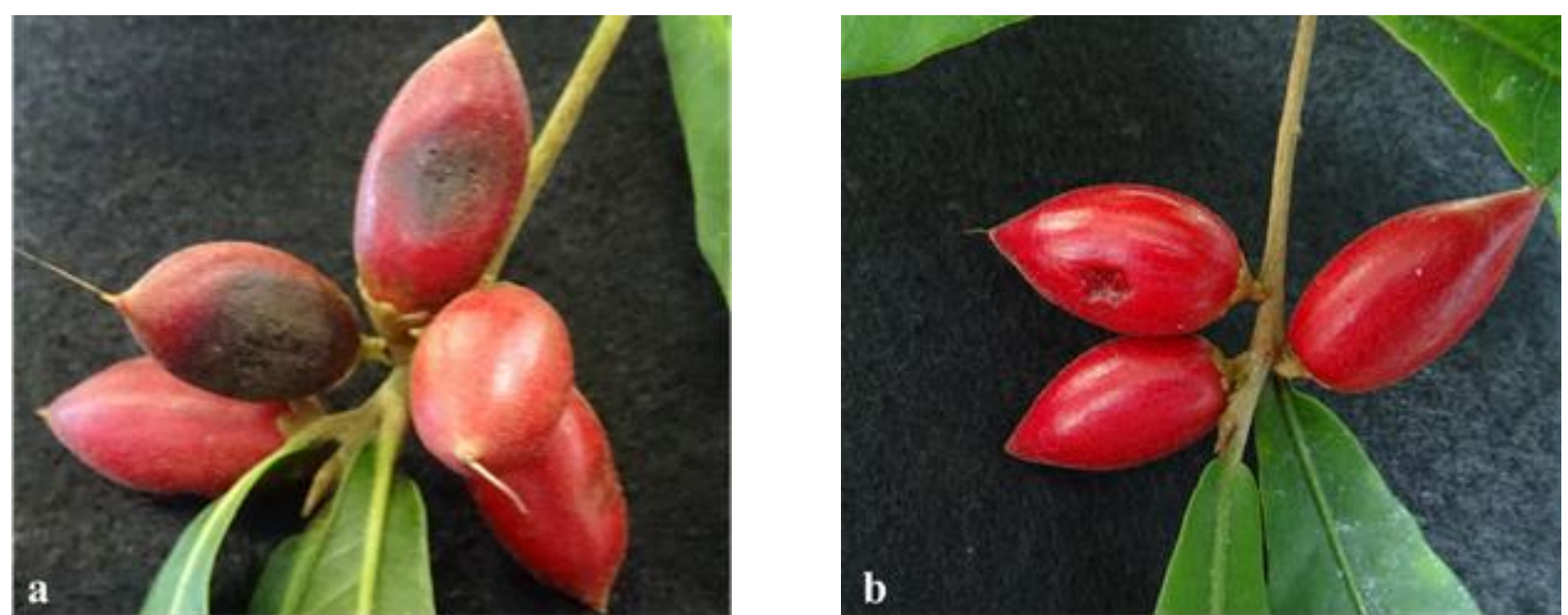

Figure 2. Fruits of S. dulcificum inoculated with strain MAFF 246750 after seven days (a) and control fruits (b).

The symptoms were first medium brown to dark brown on wounded area and then enlarged on the rest of the leaves (Figure 3 and 4). Both inoculated fungi were reisolated from the symptoms. On the control and nonwounded leaf, both MAFF 246751 and MAFF 245966 did not provide any symptom.

Phylogenetic analyses of the combined datasets: Sequence similarity searches of ITS region using BLAST were performed to identify Colletotrichum isolates. Comparisons of ITS sequences of isolates from $S$. dulcificum with sequences in GeneBank showed that

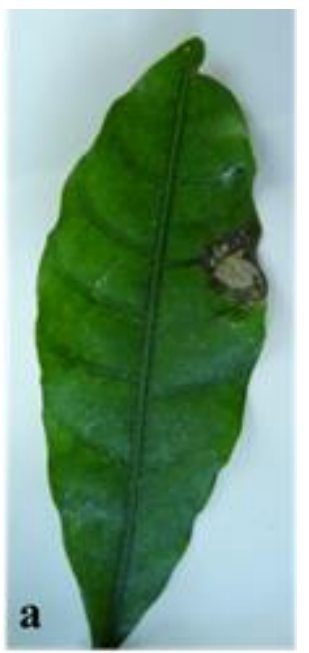

b

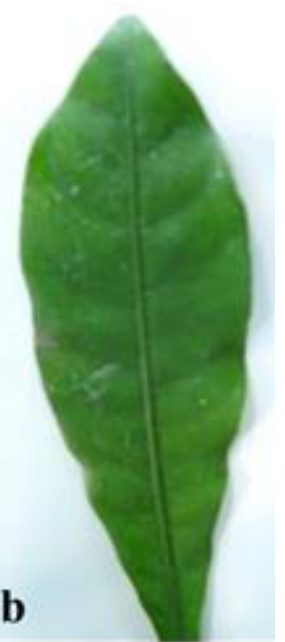

Figure 3. Pathogenicity assay of MAFF 245966 on leaves of $S$. F dulcificum after seven days. a: Inoculated leaf. b: Control leaf.

DNA sequences we used for the C. gloeosporioides species complex tree were concatenated to form a matrix of 2616 bp. The locus boundaries in the alignment were ITS:1551, GAPDH: 552-836, CAL: 837-1605, ACT: 1606-1907,
MAFF 246750 and MAFF 246751 belong to the Colletotrichum gloeosporioides species complex while MAFF 245966 belongs to the Colletotrichum boninense species complex (Data not shown). Because the two species complexes are phylogenetically diverse groups, we carried out separate phylogenetic analyses of the two species complexes as follows. C. hippeastri and $C$. boninense were selected as outgroup for the Colletotrichum gloeosporioides species complex tree, $C$. gloeosporioides for the Colletotrichum boninense species complex tree.
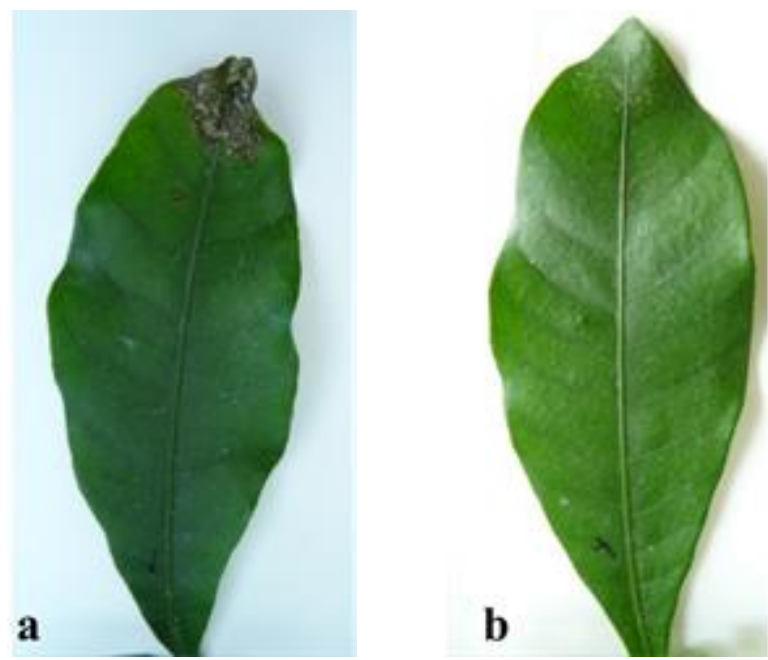

Figure 4. Pathogenicity assay of MAFF 246751 on leaves of $S$. dulcificum after seven days. a: Inoculated leaf. b: Control leaf.

and TUB2: 1908-2616. A phylogenetic analysis of the $C$. gloeosporioides species complex showed that MAFF 246750 from fruit rot and MAFF 246751 from leaf blight were clearly separated from each other (Figure 5). 


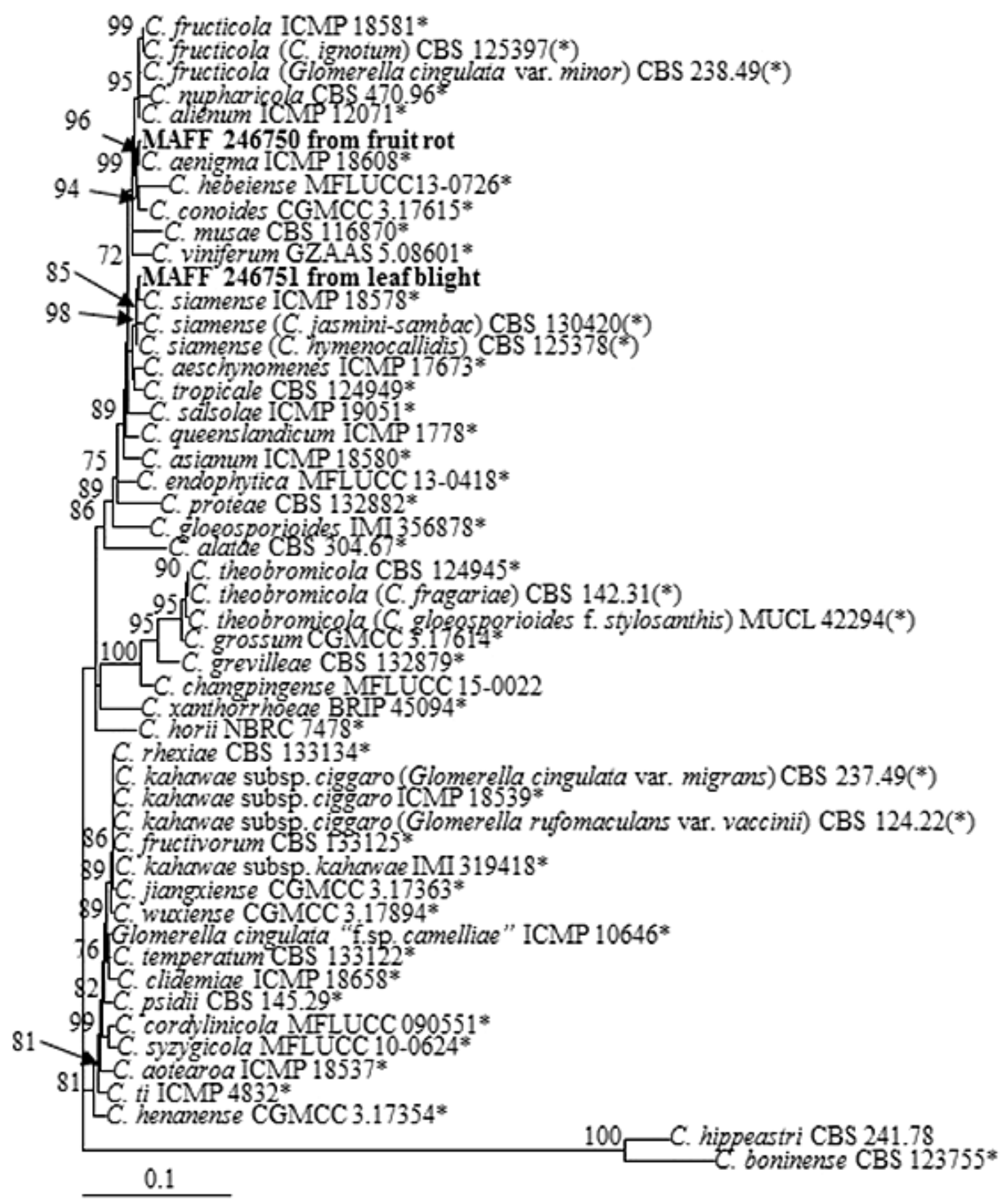

Figure 5. ML phylogenetic analysis of ITS, GAPDH, CAL, ACT and TUB2 sequences for the two isolates of Colletotrichum, MAFF 246750 from fruit rot and MAFF 246751 from leaf blight on S. dulcificum. * = ex-type culture, $\left({ }^{*}\right)=$ ex-type culture of synonymized taxon.

The tree also indicated that the most closely related species to MAFF 246750 and MAFF 246751 were C. aenigma and C. siamense with $96 \%$ and $98 \%$ bootstrap support, respectively. The ITS, GAPDH, CAL, ACT, and TUB2 sequences obtained for the $C$. boninense species complex tree were concatenated to form an alignment of $2178 \mathrm{bp}$.
The locus boundaries in the alignment were ITS: 1-551, GAPDH: 552-850, CAL: 851-1307, ACT: 1308-1602, TUB2: 1603-2178. A maximum likelihood tree of the concatenated dataset is shown in Figure 6. In this tree, MAFF 245966 fell into the $C$. karstii clade supported by $100 \%$ bootstrap value. 


\section{Morphology} Colletotrichum aenigma isolated from fruit rot on $S$. dulcificum: Colonies on PDA were flat with entire edges, white to grey and cottony with scattered pale orange conidial mass near the center. On the PDA reverse side, colonies were colorless to white and black spots occurred toward center. Asexual and sexual morphology were observed on PDA after seven days. Conidia were 14.5$19.5 \times 4-6.5 \mu \mathrm{m}$ (average $16.6 \times 5.3, \mathrm{n}=30$ ) in size, straight and cylindrical with broadly round ends.

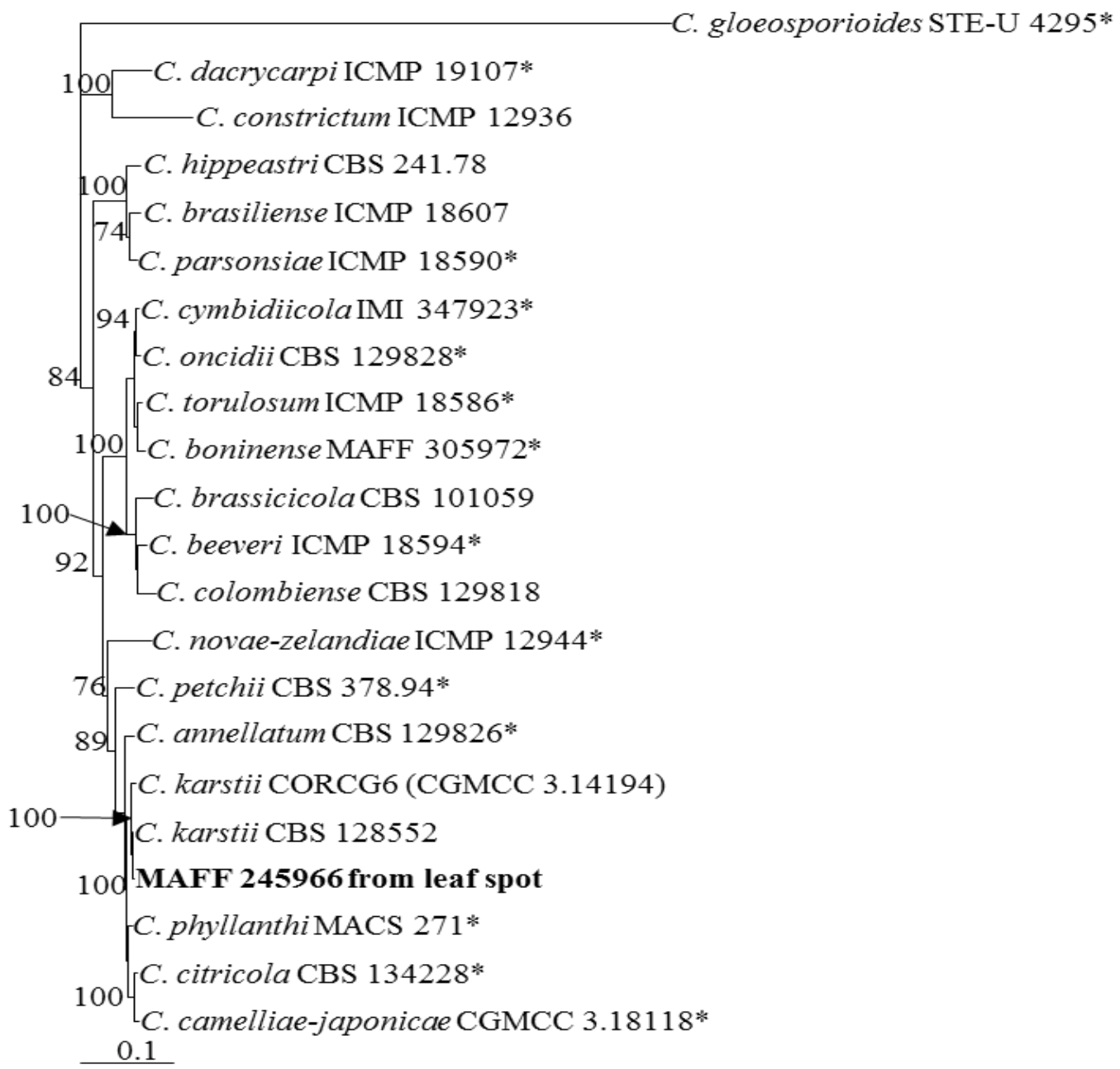

Figure 6. ML phylogenetic analysis of ITS, GAPDH, CAL, ACT and TUB2 sequences for the isolate of Colletotrichum MAFF 245966 from leaf spot on $S$. dulcificum. ${ }^{*}=$ ex-type culture.

Setae were dense, dark brown and smooth with $2-4$ septate. Appressoria were lobed and 8.5-15.0 x 5.5$9.0 \mu \mathrm{m}$ (average $11.6 \times 7.1, \mathrm{n}=30$ ) in size (Figure 7). In terms of teleomorph state, perithecia were oval and brown to dark brown color. Asci contained eight ascospores were clavate and 96.0-108.0 $\times 10-14.0 \mu \mathrm{m}$ (average $103.6 \times 12.8, \mathrm{n}=7$ ) in size. Ascospores were hyaline, aseptate, smooth, ellipsoidal and 14.0-20.0 x 4.5-8.0 $\mu \mathrm{m}$ (average $16.9 \times 6.5, \mathrm{n}=30$ ) (Figure 7) in size. These morphological characters agreed with Colletotrichum aenigma described by Weir et al. (2012).
Colletotrichum karstii isolated from leaf spot on $\boldsymbol{S}$. dulcificum: Colonies on PDA after one week at $25{ }^{\circ} \mathrm{C}$ were white to slightly grey and produced aerial mycelium at the center and scatter of tufts. On the reverse side, the colony is yellowish color near the center, colourless toward the edge. Conidia were in yellowish mass. Conidiophores were hyaline, smooth and cylindrical. Conidia on PDA plate after one week were hyaline, smooth-walled, aseptate, straight, cylindrical with broadly round ends and 14.0-18.0 x 5.5-8.0 $\mu \mathrm{m}$ in size. Setae were not observed. Appressoria on SNA were pale to medium brown, bud 
shape to bullet-shaped, smooth walled and 7.0-12.0 $\mathrm{x}$ 3.5-9.0 $\mu \mathrm{m}$ (average $8.7 \times 5.6, \mathrm{n}=30$ ) in size. Asci were unitunicate, clavate-shaped, tapering, smooth walled, 51.0-70.0 x 9.0-14.0 $\mu \mathrm{m}$ (average $59.8 \times 11.8, \mathrm{n}=7$ ) in size and contained eight ascospores. Ascospores were aseptate, hyaline, smooth walled, fusiform to ovoid, slightly curved with rounded ends, and 15.0-17.0 x 5.0$6.0 \mu \mathrm{m}$ (average $17.0 \times 6.2, \mathrm{n}=30$ ) in size (Figure 8). These morphological characters agreed with Colletotrichum karstii (Yang et al., 2011), with the exception of color of conidial mass and the shape of ascospores.
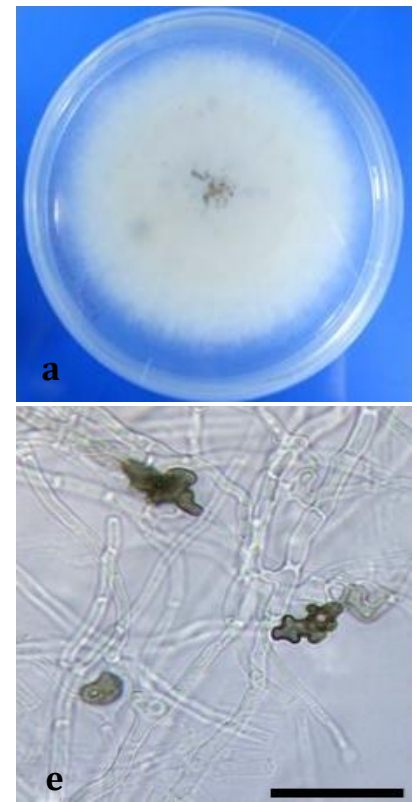
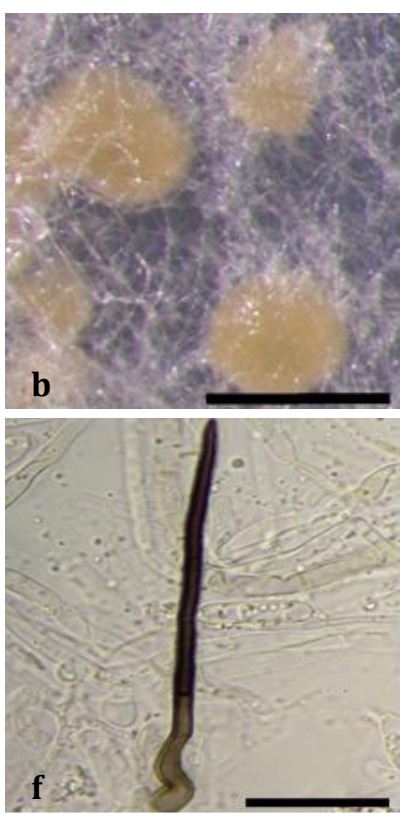
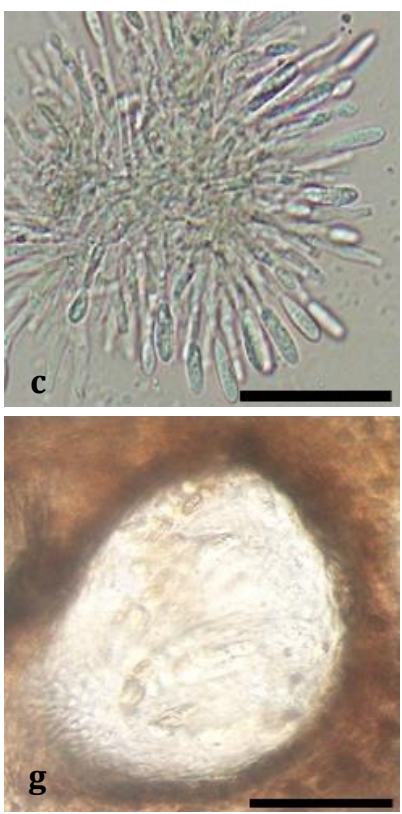
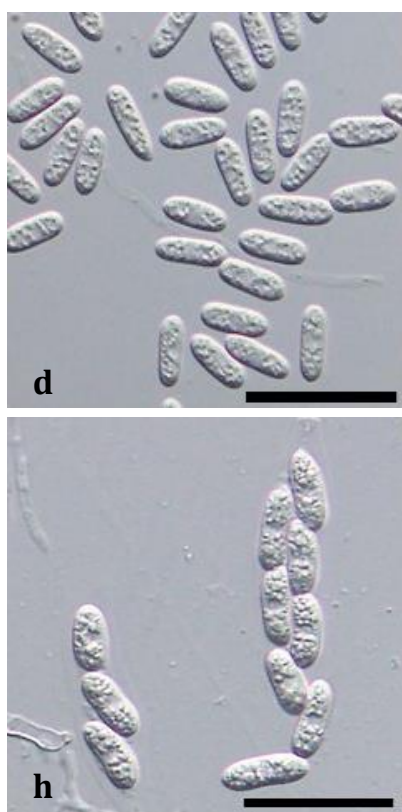

Figure 7. Colletotrichum aenigma. a: Colony on PDA after seven days (reverse). b: Conidial masses. c: Conidiophores. d: Conidia. e: Appressoria. f: Seta. g: Perithecium. h: Ascospores. Scale bars $\mathbf{b}=200 \mu \mathrm{m}$. c, d, $\mathbf{g}, \mathbf{h}=50 \mu \mathrm{m}$. e, f $=20 \mu \mathrm{m}$.
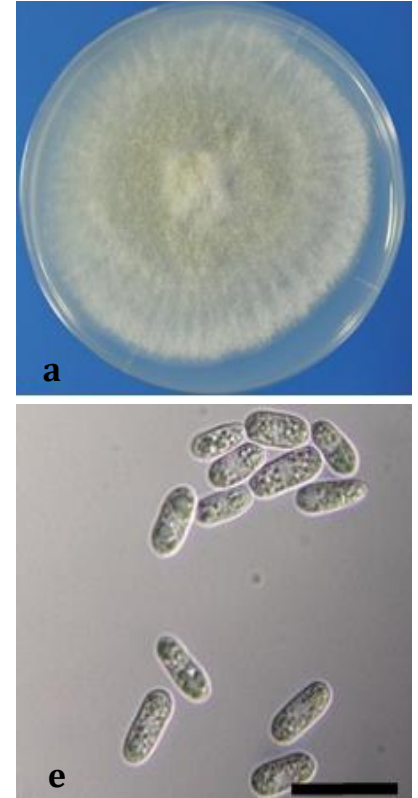
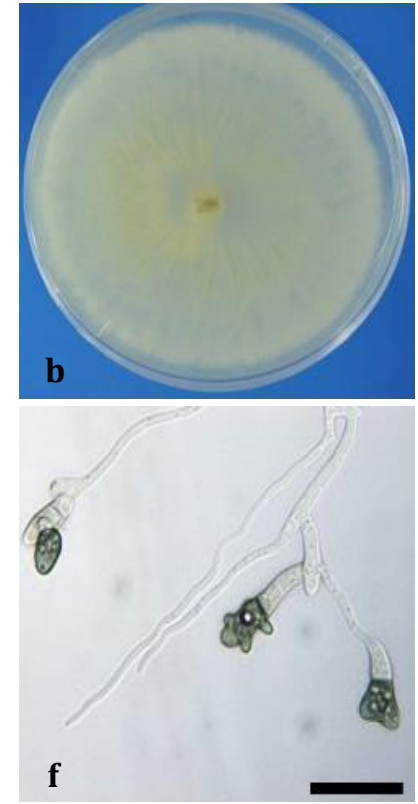
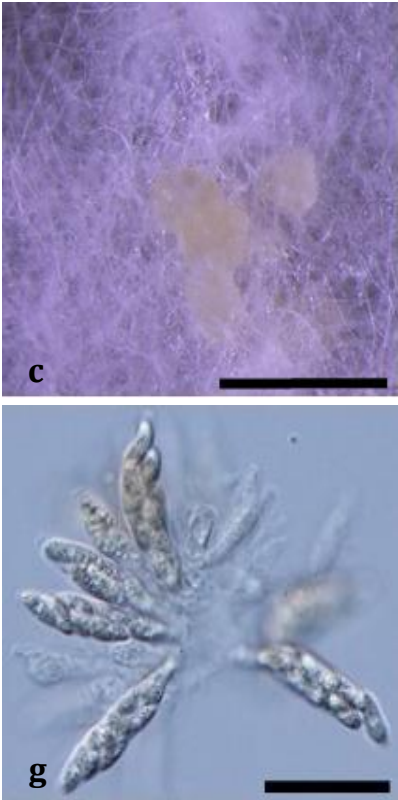

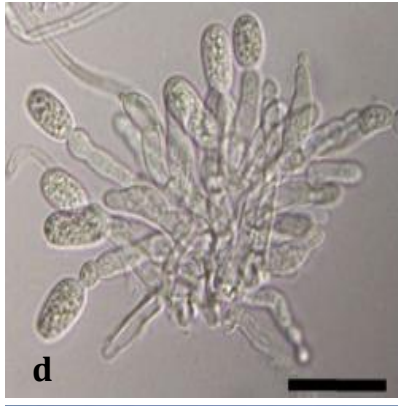

h

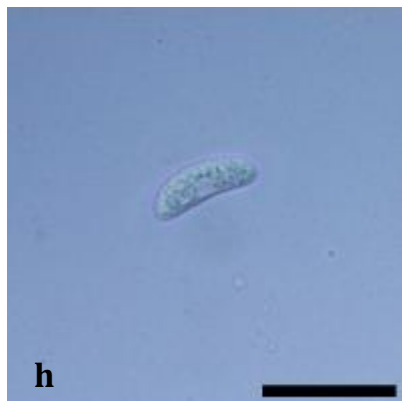

Figure 8. Colletotrichum karstii. a: Colony on PDA after seven days (surface). b: Colony on PDA for seven days (reverse). c: Conidial mass. d: Conidiophores. e: Conidia. f: Appressoria. g: Asci. h: Ascospore. Scale bars: c $=200 \mu \mathrm{m}$. d, e, f, $\mathbf{h}=$ $20 \mu \mathrm{m} . \mathbf{g}=50 \mu \mathrm{m}$. 


\section{Colletotrichum siamense isolated from leaf blight} on S. dulcificum: Colonies on PDA after seven days were white, and reverse side was pale pink. Aerial mycelium was greyish white, dense and cottony. Conidial masses were in medium to dark orange at the inoculum point (Figure 9). Setae present, 3-5 septates, pale brown to dark brown and smooth walled. Conidiophores were hyaline, smooth and cylindrical.
Conidia were one-celled, smooth-walled, hyaline with obtuse to slight rounded ends and 13.0-19.0 x 3.0-5.5 $\mu \mathrm{m}$ (average $16.3 \times 4.4$ ) in size. Appressoria were brown, ovoid, bud-shaped, and 6.0-9.5 x 4.0-6.0 $\mu \mathrm{m}$ (average $=7.9 \times 5.0, \mathrm{n}=30$ ) in size. The mycelium produced appressoria on SNA at fifth day. Teleomorph of this fungus did not produce under any condition we used.
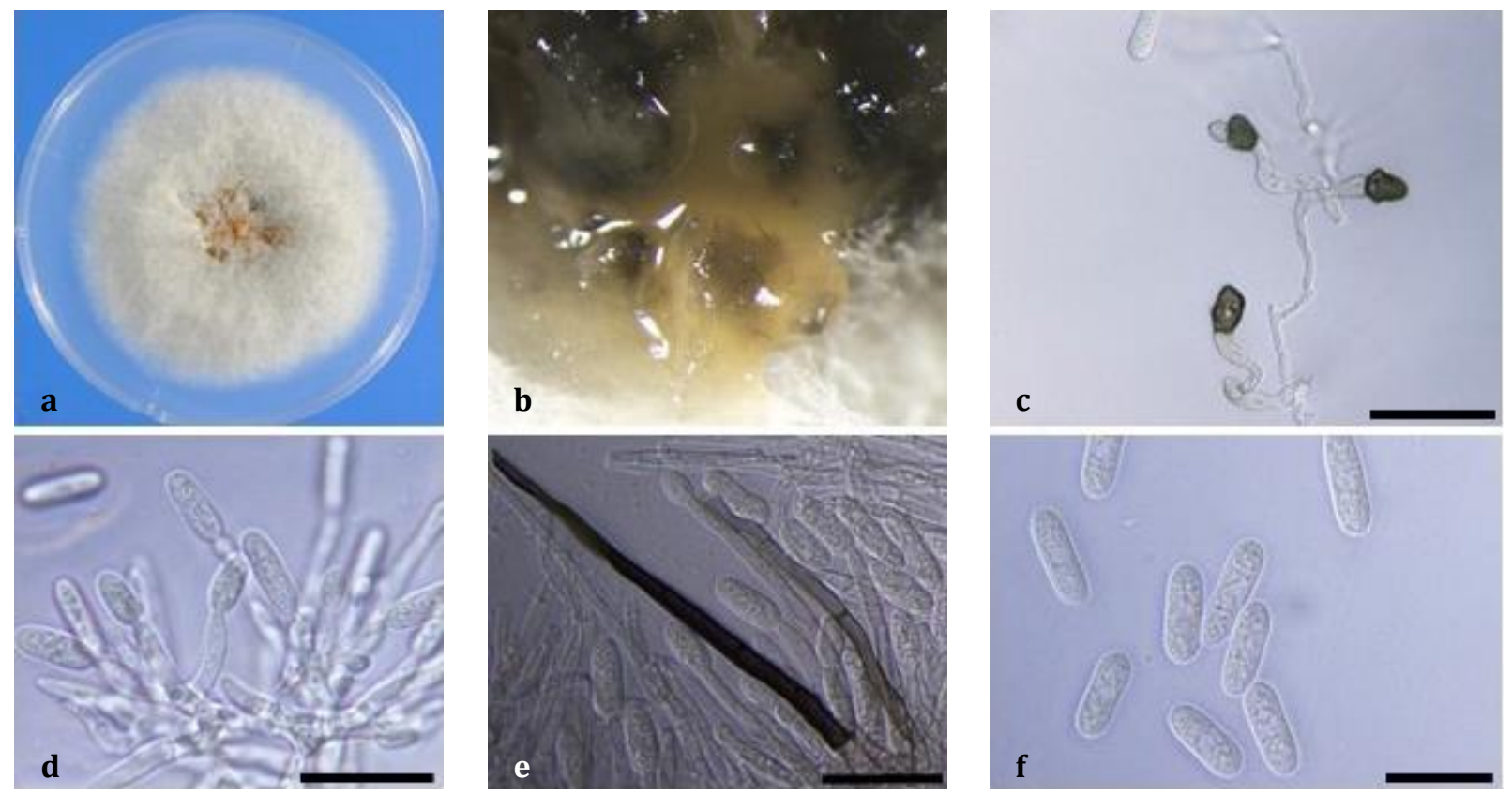

Figure 9. Colletotrichum siamense: a. Colony on PDA after seven days (surface). b: Conidial mass. c: Appressoria. d: Conidiophores. e: Setae. f: Conidia. Scale bars: c, d, e, $\mathbf{f}=20 \mu \mathrm{m}$.

\section{DISCUSSION}

Using ITS is useful in preliminary identification of fungi (Schoch et al., 2012). Our results of BLAST search indicated that our three Colletotrichum species belonged to the $C$. gloeosporioides species complex and the $C$. boninense species complex, respectively.

The fungus isolated from fruit rot was identified as Colletotrichum aenigma. This species has been reported as an anthracnose pathogen on several plants around the world (Diao et al., 2017; Gan et al., 2016; Meetum et al., 2015; Schena et al., 2013; Wang et al., 2016). Database of plant diseases in Japan (http://www.gene.affrc.go.jp /databases-micro_pl_diseases_en.php) showed $C$. aenigma to be associated with anthracnose or other diseases on Buckwheat, Japanese horse chestnut, mango, apple, melon, grape and strawberry. It suggests that this species has a wide geographic distribution and broad host range in Japan. Two isolations obtained from leaf spot and leaf blight were identified as $C$. karstii and $C$. siamense, respectively. Colletotrichum karstii has the broadest geographical range in $C$. boninense species complex (Damm et al., 2012). Our study is the second record finding $C$. karstii in Japan after Ichinose et al. (2016). This species has been found on various host plants (Lima et al., 2013). Damm et al. (2012) identified culture strain CBS 128552 found on leaf of Synsepalum dulcificum as C. karstii. However, the pathogenicity of this species on S. dulcificum has not been tested before. Based on the result of our study, we found that this species causes of leaf spot on S. dulcificum. Colletotrichum siamense belonging to the $C$. gloeosporioides species complex was first confirmed as pathogen associated with anthracnose of coffee berries in the northern Thailand (Prihastuti et al., 2009), and this species has now been recorded on many hosts (Honger et al., 2016; Sharma and Shenoy, 2013). It is evaluated as a dominant species on 
tropical fruits (Sharma and Shenoy, 2013). Recently the taxonomic position of $C$. siamense has been under debate. Prihastuti et al. (2009) and Wikee et al. (2010) found $C$. siamense could be a species complex whereas Liu et al. (2016) indicated $C$. siamense as a single species based on statistical analysis using multi-locus sequence data, cross-mating and genetic recombination test. In this study, our phylogenetic tree showed slight phylogenetic distance between our isolate and C. siamense supported by $98 \%$ bootstrap value. We therefore tentatively identified it as $C$. siamense.

This study provides the first report of fruit rot, leaf blight and leaf spot caused by three Colletotrichum species on $S$. dulcificum based on pathogenicity test, morphological and molecular identification methods. This information of host and pathogen will aid plant pathologists in designing disease control strategies for S. dulcificum. Further studies such as host range, disease impact on yield, and control methods for these Colletotrichum species above are required to protect $S$. dulcificum from anthracnose.

\section{ACKNOWLEDGEMENT}

We sincerely appreciate Shigeki Hayashi (Research Center for Medicinal Plant Resources), Naoko Anjiki (Research Center for Medicinal Plant Resources), Hiromichi Horie (Hosei University) and Kaoru Minoguchi (Ofuna Botanical Garden) each of whom contributed to our various collecting trips. We would also like to thank all our colleagues at Hosei University.

\section{REFERENCES}

Akinmoladun, A. C. 2016. Effect of Synsepalum dulcificum berry extract on oxidative stress and hepatotoxicity indices, following subacute administration in normal rats. FUTA Journal of Research in Sciences, 12: 167-177.

Alizadeh, A., M. Javan-Nikkhah, R. Zare, K. B. Fotouhifar, U. Damm and E. H. Stukenbrock. 2015. New records of Colletotrichum species for the mycobiota of Iran. Mycologia Iranica, 2: 95-109.

Cai, L., K. D. Hyde, P. W. J. Taylor, B. Weir, J. Waller, M. M. Abang, J. Z. Zhang, Y. L. Yang, S. Phoulivong and Z. Y. Liu. 2009. A polyphasic approach for studying Colletotrichum. Fungal Diversity, 39: 183-204.

Carbone, I. and L. M. Kohn. 1999. A method for designing primer sets for speciation studies in filamentous ascomycetes. Mycologia, 91: 553-556.

Chen, Y., G. Wei and W. Chen. 2002. New species of Pestalotiopsis. Mycosystema, 21: 316-323.
Da Silva, C. d. F. B. and S. J. Michereff. 2013. Biology of Colletotrichum spp. and epidemiology of the anthracnose in tropical fruit trees. Revista Caatinga, 26: 130-138.

Damm, U., P. F. Cannon, J. H. C. Woudenberg, P. R. Johnston, B. S. Weir, Y. P. Tan, R. G. Shivas and P. W. Crous. 2012. The Colletotrichum boninense species complex. Studies in Mycology, 73: 1-36.

De Silva, D. D., P. K. Ades, P. W. Crous and P. W. J. Taylor. 2016. Colletotrichum species associated with chili anthracnose in Australia. Plant Pathology, 66: 254267.

Dean, R., J. A. L. Van Kan, Z. A. Pretorius, K. E. HammondKosack, A. Di Pietro, P. D. Spanu, J. J. Rudd, M. Dickman, R. Kahmann and J. Ellis. 2012. The top 10 fungal pathogens in molecular plant pathology. Molecular Plant Pathology, 13: 414-430.

Diao, Y. Z., C. Zhang, F. Liu, W. Z. Wang, L. Liu, L. Cai and X. L. Liu. 2017. Colletotrichum species causing anthracnose disease of chili in China. PersooniaMolecular Phylogeny and Evolution of Fungi, 38: 20-37.

Gan, P., N. Nakata, T. Suzuki and K. Shirasu. 2016. Markers to differentiate species of anthracnose fungi identify Colletotrichum fructicola as the predominant virulent species in strawberry plants in Chiba Prefecture of Japan. Journal of General Plant Pathology, 83: 14-22.

Gardes, M. and T. D. Bruns. 1993. ITS primers with enhanced specificity for basidiomycetesapplication to the identification of mycorrhizae and rusts. Molecular Ecology, 2: 113-118.

Guerber, J. C., B. Liu, J. C. Correll and P. R. Johnston. 2003. Characterization of diversity in Colletotrichum acutatum sensu lato by sequence analysis of two gene introns, mtDNA and intron RFLPs, and mating compatibility. Mycologia, 95: 872-885.

Honger, J. O., S. K. Offei, K. A. Oduro, G. T. Odamtten and S. T. Nyaku. 2016. Identification and molecular characterisation of Colletotrichum species from avocado, citrus and pawpaw in Ghana. South African Journal of Plant and Soil, 33: 177-185.

Ichinose, R., H. Hoshi, T. Ono, Y. Hirooka and H. Horie. 2016. Anthracnose diseases found on Aesculus indica, Cornus kousa and Acer platanoides in Japan. Annual report of the kanto-tosan plant protection society. 63-123.

Lima, N. B., M. V. de A. Batista, M. A. De Morais, M. A. G. Barbosa, S. J. Michereff, K. D. Hyde and M. P. S. 
Câmara. 2013. Five Colletotrichum species are responsible for mango anthracnose in northeastern Brazil. Fungal Diversity, 61: 75-88.

Liu, F., M. Wang, U. Damm, P. W. Crous and L. Cai. 2016. Species boundaries in plant pathogenic fungi: A Colletotrichum case study. BMC Evolutionary Biology, 16: 81-86.

Maddison, W. P. 2017. Mesquite: A modular system for evolutionary analysis; 2007. Version 3.2. http://mesquiteproject.org.

Meetum, P., C. Leksomboon and M. Kanjanamaneesathian. 2015. First report of Colletotrichum aenigma and $C$. siamense, the causal agents of anthracnose disease of dragon fruit in Thailand. Journal of Plant Pathology, 97: 402.

Nguyen, P. T. H., O. Vinnere Pettersson, P. Olsson and E. Liljeroth. 2009. Identification of Colletotrichum species associated with anthracnose disease of coffee in Vietnam. European Journal of Plant Pathology, 127: 73-87.

O'Donnell, K. and E. Cigelnik. 1997. Two divergent intragenomic rDNA ITS2 types within a monophyletic lineage of the fungus Fusarium are nonorthologous. Molecular Phylogenetics and Evolution, 7: 103-116.

O'Donnell, K., H. I. Nirenberg, T. Aoki and E. Cigelnik. 2000. A multigene phylogeny of the Gibberella fujikuroi species complex: Detection of additional phylogenetically distinct species. Mycoscience, 41: 61-78.

Prihastuti, H., L. Cai, H. Chen, E. H. C. McKenzie and K. D. Hyde. 2009. Characterization of Colletotrichum species associated with coffee berries in northern Thailand. Fungal Diversity, 39: 89-109.

Rodrigues, J. F., R. d. S. Andrade, S. C. Bastos, S. B. Coelho and A. C. M. Pinheiro. 2016. Miracle fruit: An alternative sugar substitute in sour beverages. Appetite, 107: 645-653.

Rojas, E. I., S. A. Rehner, G. J. Samuels, S. A. Van Bael, E. A. Herre, P. Cannon, R. Chen, J. Pang, R. Wang, Y. Zhang, Y.-Q. Peng and T. Sha. 2010. Colletotrichum gloeosporioides s.l. associated with Theobroma cacao and other plants in Panamá: Multilocus phylogenies distinguish host-associated pathogens from asymptomatic endophytes. Mycologia, 102: 1318-1338.

Schena, L., S. Mosca, S. O. Cacciola, R. Faedda, S. M. Sanzani, G. E. Agosteo, V. Sergeeva and G. Magnano di San Lio. 2013. Species of the Colletotrichum gloeosporioides and C.boninense complexes associated with olive anthracnose. Plant Pathology, 63: 437-446.

Schoch, C. L., K. A. Seifert, S. Huhndorf, V. Robert, J. L. Spouge, C. A. Levesque, W. Chen, E. Bolchacova, K. Voigt and P. W. Crous. 2012. Nuclear ribosomal internal transcribed spacer (ITS) region as a universal DNA barcode marker for Fungi. Proceedings of the National Academy of Sciences, 109: 6241-6246.

Sharma, G. and B. D. Shenoy. 2013. Colletotrichum fructicola and C. siamense are involved in chilli anthracnose in India. Archives of Phytopathology and Plant Protection, 47: 1179-1194.

Sharma, G. and B. D. Shenoy. 2016. Colletotrichum systematics: Past, present and prospects. Mycosphere, 7: 1093-1102.

Shi, Y.-C., K.-S. Lin, Y.-F. Jhai, B.-H. Lee, Y. Han, Z. Cui, W.-H. Hsu and S.-C. Wu. 2016. Miracle Fruit (Synsepalum dulcificum) exhibits as a novel anti-hyperuricaemia agent. Molecules, 21: 140-144.

Stamatakis, A., P. Hoover and J. Rougemont. 2008. A rapid bootstrap algorithm for the RAxML web servers. Systematic Biology, 57: 758-771.

Wang, Y.-C., X.-Y. Hao, L. Wang, X. Bin, X.-C. Wang and Y.J. Yang. 2016. Diverse Colletotrichum species cause anthracnose of tea plants (Camellia sinensis (L.) 0. Kuntze) in China. Scientific Reports, 6: 35287.

Weir, B. S., P. R. Johnston and U. Damm. 2012. The Colletotrichum gloeosporioides species complex. Studies in Mycology, 73: 115-180.

Wikee, S., L. Cai, N. Pairin, E. H. C. McKenzie, Y.-Y. Su, E. Chukeatirote, H. N. Thi, A. H. Bahkali, M. A. Moslem, K. Abdelsalam and K. D. Hyde. 2010. Colletotrichum species from Jasmine (Jasminum sambac). Fungal Diversity, 46: 171-182.

Yang, Y., L. Cai, Z. Yu, Z. Liu and K. D. Hyde. 2011. Colletotrichum Species on Orchidaceaein Southwest China. Cryptogamie Mycologie, 32: 229-253.

Yokosawa, S., N. Eguchi, K.-i. Kondo and T. Sato. 2017. Phylogenetic relationship and fungicide sensitivity of members of the Colletotrichum gloeosporioides species complex from apple. Journal of General Plant Pathology, 83: 291-298.

Zivkovic, S., S. Stojanovic, Z. Ivanovic, N. Trkulja, N. Dolovac, G. Aleksic and J. Balaz. 2010. Morphological and molecular identification of Colletotrichum acutatum from tomato fruit. Pesticidi i Fitomedicina, 25: 231-239. 
Publisher's note: EScience Press remains neutral with regard to jurisdictional claims in published maps and institutional affiliations.

(c) (i)

Open Access This article is licensed under a Creative Commons Attribution 4.0 International License, which permits use, sharing, adaptation, distribution and reproduction in any medium or format, as long as you give appropriate credit to the original author(s) and the source, provide a link to the Creative Commons license and indicate if changes were made. The images or other third-party material in this article are included in the article's Creative Commons license, unless indicated otherwise in a credit line to the material. If material is not included in the article's Creative Commons license and your intended use is not permitted by statutory regulation or exceeds the permitted use, you will need to obtain permission directly from the copyright holder. To view a copy of this license, visit http://creativecommons.org/licenses/by/4.0/.

(C) The Author(s) 2018. 